Pacific Journal of Mathematics

A PHRAGMÉN-LINDELÖF THEOREM

T. LANG AND Y.W. LU 


\title{
A PHRAGMÉN-LINDELÖF THEOREM
}

\author{
X. T. LIANG AND Y. W. LU
}

Let $\Omega$ be an unbounded and connected domain in $E^{n}$. Consider on $\Omega \times(0, \infty)$ the parabolic equation

$$
u_{t}-\operatorname{div} \mathbf{A}(x, t, u, \nabla u)=B(x, t, u, \nabla u) .
$$

Under proper conditions a theorem of Phragmén-Lindelöf type is proved for generalized solutions of the equation.

Introduction. The classical Phragmén-Lindelöf principle gives an important property of harmonic functions defined on a plane sector domain. That has been generalized not only to generalized solutions of quasi-linear elliptic equations in more general unbounded and connected domains (see [1]-[5]), but also to the ones of quasilinear parabolic equations in divergence form which have their principal parts only [6]. In this paper the result is extended to generalized solutions of the equation (1). We prove the result by an argument based on the technique of Moser [7] and Ladyženskaja-Ural'ceva [8]. We have not seen any reference discussing such behavior for solutions of parabolic equations except [6] where the simpler situation of the equation (1), namely $B \equiv 0$, is considered.

The paper is organized as follows. In $\S 1$ the main result is mentioned and in $\S 2$ several lemmata are given as preliminaries. Finally, a full proof of our theorem is stated in $\S 3$.

1. Main result. Let $\Omega$ be an unbounded and connected domain in the $n$-dimensional Euclidean space $E^{n}$. Denote by $\partial \Omega$ the boundary of $\Omega$. On $\Omega \times(0, \infty)$ we consider the following equation:

$$
u_{t}-\operatorname{div} \mathbf{A}(x, t, u, \nabla u)=B(x, t, u, \nabla u)
$$

where $A(x, t, u, \xi)$ and $B(x, t, u, \xi)$ are defined on $\Omega \times(0, \infty) \times$ $E^{1} \times E^{n}$, continuous with respect to $u$ and $\xi$ for fixed $x$ and $t$, measurable with respect to $x$ and $t$ for fixed $u$ and $\xi$, and satisfying the following structural conditions:

$$
\begin{aligned}
\xi \cdot \mathbf{A}(x, t, u, \xi) & \geq \kappa_{0}|\xi|^{2} \\
|\mathbf{A}(x, t, u, \xi)| & \leq \kappa_{1}|\xi| \\
|B(x, t, u, \xi)| & \leq b(x, t)|\xi|
\end{aligned}
$$


where $\kappa_{1} \geq \kappa_{0}>0, b(x, t) \in L_{\infty}(\Omega \times(0, \infty))$ and

$$
|b(x, t)|=O\left(|x|^{-1}\right) \text { (uniformly for } t \text { ) as }|x| \rightarrow \infty .
$$

We need the supposition on $\Omega$ : there exist some $x_{0} \in \partial \Omega$ and a $\theta \in(0,1)$ such that

$$
\begin{aligned}
& \operatorname{meas}\left(\Omega \cap\left\{B\left(x_{0}, \rho_{0}\right) \backslash B\left(x_{0}, \rho_{1}\right)\right\}\right) \\
& \leq \theta \operatorname{meas}\left\{B\left(x_{0}, \rho_{0}\right) \backslash B\left(x_{0}, \rho_{1}\right)\right\}
\end{aligned}
$$

for any $\rho_{0}>\rho_{1}>0$, where meas $e$ denotes the Lebesgue measure of the set $e$ in $E^{n}$ and

$$
B\left(x_{0}, \rho\right)=\left\{x \in E^{n},\left|x-x_{0}\right|<\rho\right\} .
$$

For $G \subset E^{n}, W_{2}^{1}(G)$ and $\stackrel{\circ}{W}_{2}^{1}(G)$ stand for the usual Sobolev spaces. Let $X$ be a Banach space formed by measurable functions defined on $G$ with respect to the norm $\|\cdot\|_{X}$. Denote $L_{p}(0, T, X)$ the Banach space formed by the mapping from $[0, T]$ into $X$ with norm $\|u\|_{L_{p}(0, T, X)}$ defined by

$$
\|u\|_{L_{p}(0, T, X)}=\left(\int_{0}^{T}\|u\|_{X}^{p} d x\right)^{1 / p} \quad\left(=\underset{t \in(0, T)}{\operatorname{ess} \sup }\|u\|_{X} \text { if } p=\infty\right) .
$$

Similarly, the space $C(0, T, X)$ etc. can also be defined.

The function $u$ is called a generalized solution of the equation (1) if for any $T>0$ and for arbitrary $G \subset \Omega$ and $G \subset \subset E^{n}$,

$$
u \in C\left(0, T, L_{2}(G)\right) \cap L_{2}\left(0, T, W_{2}^{1}(G)\right)
$$

and the following holds:

$$
\begin{aligned}
(1)^{\prime} \int_{0}^{t} \int_{G}\left\{-v_{t} u+\nabla v \cdot A(x, t, u, \nabla u)\right. & -v B(x, t, u, \nabla u)\} d x d t \\
& +\left.\int_{G} v(x, t) u(x, t)\right|_{t=0} ^{t=t} d x=0,
\end{aligned}
$$

$$
\forall t \in(0, T), \quad v \in W_{2}^{1}\left(0, T, L_{2}(G)\right) \cap L_{2}\left(0, T, \stackrel{\circ}{W}_{2}^{1}(G)\right)
$$

where $u(x, 0)$ is a given initial value of $u$.

As the main result we have

TheOREM. Suppose that the conditions (2)-(4) are satisfied and the generalized solution $u$ of the equation (1) satisfies

$$
u^{+}=\max (u, 0)=0 \quad \text { on } \partial \Omega \times(0, \infty) \text { and }\left.u^{+}\right|_{t=0}=0 \text {. }
$$


If there exists an $R>0$ such that $M(R)>0$, then

$$
M(\rho) \rightarrow \infty \text { as } \rho \rightarrow \infty
$$

where

$$
M(\rho)=\underset{Q(\rho)}{\operatorname{ess} \sup } u(x, t), \quad Q(\rho)=\left\{\Omega \cap B\left(x_{0}, \rho\right)\right\} \times\left(0, \rho^{2}\right) .
$$

As an immediate consequence we have

COROLLARY. If the $u$ in the theorem is bounded from above, then $u \leq 0$ on $\Omega \times(0, \infty)$.

REMARK. The results of the theorem and corollary and the proof given in $\S 3$ below are also true for subsolutions of the equation (1). As the definition $u$ is a subsolution if besides (5) it satisfies the following:

$$
\begin{array}{r}
\int_{t^{\prime}}^{t^{\prime \prime}} \int_{G}\left\{-v_{t} u+\nabla v \cdot \mathbf{A}(x, t, u, \nabla u)-v B(x, t, u, \nabla u)\right\} d x d t \\
\quad+\left.\int_{G} v(x, t) u(x, t)\right|_{t=t^{\prime}} ^{t=t^{\prime \prime}} d x \leq 0, \\
\forall\left(t^{\prime}, t^{\prime \prime}\right) \subset(0, T), \quad v \in W_{2}^{1}\left(0, T, L_{2}(G)\right) \cap L_{2}\left(0, T, \stackrel{\circ}{W}_{2}^{1}(G)\right) \\
\text { and } v \geq 0 .
\end{array}
$$

\section{Preliminaries.}

Lemma 1. Suppose $G$ is a bounded domain in $E^{n}, T>0$ is a definite value and $u$ satisfies (5) and (1)'. If there exists a constant $M>0$ such that

$$
(u-M)^{+} \in L_{2}\left(0, T, \stackrel{\circ}{W} \frac{1}{2}(G)\right) \text { and }\left.(u-M)^{+}\right|_{t=0}=0
$$

then

$$
\underset{G \times(0, T)}{\operatorname{ess} \sup } u(x, t) \leq M .
$$

Proof. If the statement were not true, there would be a

$$
M^{\prime}=\underset{G \times(0, T)}{\operatorname{ess} \sup } u>M \quad\left(M^{\prime}=\infty \text { is not exclusive }\right) .
$$

By (7), we have for any $k \in\left(M, M^{\prime}\right)$

$$
(u-k)^{+} \in L_{2}\left(0, T, \stackrel{\circ}{W} \frac{1}{2}(G)\right) \text { and }\left.(u-k)^{+}\right|_{t=0}=0 .
$$


Hence it follows by the imbedding inequality in $L_{2}\left(0, T, \stackrel{\circ}{W}_{2}^{1}(G)\right)$ that

$$
\left(\int_{0}^{T} \int_{G}\left|(u-k)^{+}\right|^{q} d x d t\right)^{2 / q} \leq C(n)||\left|(u-k)^{+}\right| \|_{G \times(0, T)}
$$

where $q=2(1+2 / n)$ and

$$
\begin{aligned}
\left\|\left|(u-k)^{+}\right|\right\|_{G \times(0, T)}= & \underset{G \times(0, T)}{\operatorname{ess} \sup _{G}} \int_{G}\left|(u-k)^{+}\right|^{2} d x \\
& +\int_{0}^{T} \int_{G}\left|\nabla(u-k)^{+}\right|^{2} d x d t .
\end{aligned}
$$

We assume temporarily that $(u-k)^{+} \in W_{2}^{1}\left(0, T, L_{2}(G)\right)$; then $v=$ $(u-k)^{+}$can be taken as a test function. Substituting $v$ into $(1)^{\prime}$ and integrating by parts with respect to $t$, we have by the use of (2) that

$$
\begin{aligned}
\int_{G}\left|(u-k)^{+}\right|^{2} d x+\int_{0}^{t} \int_{G}\left|\nabla(u-k)^{+}\right|^{2} d x d t \\
\leq C \int_{0}^{t} \int_{G} b(x, t)(u-k)^{+}\left|\nabla(u-k)^{+}\right| d x d t,
\end{aligned}
$$

where the constant $C>0$ depends only on $n$ and $\kappa_{0}$. However, we cannot guarantee $(u-k)^{+} \in W_{2}^{1}\left(0, T, L_{2}(G)\right)$ when $u$ is the function in Lemma 1. What we have to do now is to extend $(u-k)^{+}$ to $G \times(-\infty, 0)$ by letting $(u-k)^{+}=0$ and instead of $v$ we take

$$
v^{\prime}=\frac{1}{h} \int_{t}^{t+h}(u-k)^{+} d \tau
$$

as the test function. Repeating the above process again we obtain (9) by letting $h \rightarrow 0$ in the last result.

Since the two terms on the left-hand side of (9) are all non-negative, each of them does not exceed that on the right-hand side. Taking their supremums for $t \in(0, T)$, we have

$$
\|(u-k)^{+}||_{G \times(0, T)} \leq C \int_{0}^{T} \int_{G}(u-k)^{+}\left|\nabla(u-k)^{+}\right| d x d t,
$$

where we absorb the $\|b(x, t)\|_{L_{\infty}}$ into the constant $C$. Considering that the effective integral domain in (10) is only $\{G \times(0, T)\} \cap$ 
$\left\{k<u<M^{\prime}\right\}$, we then have by Hölder inequality that

$$
\begin{aligned}
& \int_{0}^{T} \int_{G}(u-k)^{+}\left|\nabla(u-k)^{+}\right| d x d t \\
& \leq \varepsilon\left(k, M^{\prime}\right)\left(\int_{0}^{T} \int_{G}\left|(u-k)^{+}\right|^{q} d x d t\right)^{1 / q} \\
& \cdot\left(\int_{0}^{T} \int_{G}\left|\nabla(u-k)^{+}\right|^{2} d x d t\right)^{1 / 2} \\
& \leq C(n) \varepsilon\left(k, M^{\prime}\right)\left|\left\|(u-k)^{+} \mid\right\|_{G \times(0, T)}\right.
\end{aligned}
$$

where

$$
\varepsilon\left(k, M^{\prime}\right)=\left(\int_{0}^{T} \int_{G \cap\left\{k<u<M^{\prime}\right\}} d x d t\right)^{1 /(n+2)} .
$$

Combining (10) with (11) we get

$$
1 \leq C(n) \varepsilon\left(k, M^{\prime}\right),
$$

where the constant $C(n)>0$ is independent of $k$. So, we have $\varepsilon\left(k, M^{\prime}\right) \rightarrow 0$ as $k \rightarrow M^{\prime}$ because

$$
\iint_{\{G \times(0, T)\} \cap\left\{k<u<M^{\prime}\right\}} d x d t \rightarrow 0 \quad \text { as } k \rightarrow M^{\prime} .
$$

Hence, the contradiction is obtained by (12).

For simplicity we write $B(\rho)=B(0, \rho)$.

Lemma 2. Suppose $\rho_{0}>\rho_{1}>0, S \subset B\left(\rho_{0}\right) \backslash B\left(\rho_{1}\right)$ and

$$
\text { meas } S \geq \theta \operatorname{meas}\left\{B\left(\rho_{0}\right) \backslash B\left(\rho_{1}\right)\right\}, \quad \theta \in(0,1) .
$$

Suppose $u \in W_{p}^{1}\left(B\left(\rho_{0}\right) \backslash B\left(\rho_{1}\right)\right), p \geq 1$ and $u=0$ on $S$. Then

$$
\int_{B\left(\rho_{0}\right) \backslash B\left(\rho_{1}\right)}|u|^{p} d x \leq C\left(n, p, \theta, \frac{\rho_{0}}{\rho_{1}}\right) \rho_{0}^{p} \int_{B\left(\rho_{0}\right) \backslash B\left(\rho_{1}\right)}|\nabla u|^{p} d x .
$$

Lemma 2 is a variety of Theorem 3.6.5, in Morrey [9] and it can be proved by the same method.

LEMMA 3 [10]. Let $f(t)$ be a non-negative bounded function defined for $0 \leq r^{\prime} \leq t \leq r$. If

$$
f(t) \leq A(s-t)^{-\alpha}+B+\theta f(s), \quad \forall r^{\prime} \leq t<s \leq r
$$


where $A, B, \alpha, \theta$ are non-negative constants and $\theta \in(0,1)$, then there exists a constant $C$ depending only on $\alpha$ and $\theta$ such that

$$
f(\rho) \leq C\left(A(R-\rho)^{-\alpha}+B\right), \quad \forall r^{\prime} \leq \rho<R \leq r .
$$

3. Proof of the theorem. Without loss of generality, let $x_{0}$ be the origin. We can rewrite the condition (3) as

$$
|b(x, t)| \leq K|x|^{-1} \text { as }|x| \geq 1,
$$

where $K$ is a positive constant.

Let $\rho \geq \max (R, 1), 0 \leq \rho_{2}<\rho_{1}<\rho_{0} \leq \rho$ and let $\zeta(x)=\zeta(|x|)$ be a piecewise linear and continuous function of $|x|$ satisfying

$$
\zeta(x)= \begin{cases}0, & \text { as }|x| \leq 2 \rho-\rho_{1} \text { or }|x| \geq 4 \rho+\rho_{1}, \\ 1, & \text { as } 2 \rho-\rho_{2} \leq|x| \leq 4 \rho+\rho_{2} .\end{cases}
$$

Then

$$
|\nabla \zeta(x)| \leq\left(\rho_{1}-\rho_{2}\right)^{-1} .
$$

The function $u$ in the theorem as the generalized solution satisfying (5) and (6) is locally bounded from above on $(\Omega \cup \partial \Omega) \times(0, \infty)$ [11]. Therefore

$$
M(\rho)=\underset{Q(\rho)}{\operatorname{ess} \sup } u(x, t)<\infty, \quad Q(\rho)=\{\Omega \cap B(\rho)\} \times\left(0, \rho^{2}\right) .
$$

On $Q(5 \rho)$ let

$$
\begin{aligned}
& w(x, t)=\ln \frac{M(5 \rho)+\varepsilon}{M(5 \rho)+\varepsilon-u^{+}}, \quad \varepsilon>0, \\
& v(x, t)=\frac{\zeta^{2}(x)(w-k)^{+}}{M(5 \rho)+\varepsilon-u^{+}}, \quad k \geq 0 .
\end{aligned}
$$

Because of the boundedness of $u$ on $Q(5 \rho)$, we have

$$
\begin{aligned}
& w \in L_{2}\left(0,25 \rho^{2} \cdot W_{2}^{1}(\Omega \cap B(5 \rho)) \cap L_{\infty}(Q(5 \rho)),\right. \\
& w=0 \text { on }\{\partial \Omega \cap B(5 \rho)\} \times\left(0,25 \rho^{2}\right) \cup\{t=0\}
\end{aligned}
$$

and

$$
v \in L_{2}\left(0,25 \rho^{2}, \stackrel{\circ}{W}_{2}^{1}(\Omega \cap B(5 \rho))\right) \cap L_{\infty}(Q(5 \rho)),\left.\quad v\right|_{t=0}=0 .
$$

Suppose $v \in W_{2}^{1}\left(0,25 \rho^{2}, L_{2}(\Omega \cap B(5 \rho))\right)$ (otherwise, we add a limit process to arrive at the same result). Such $v$ can be taken as a test 
function. Substituting it into $(1)^{\prime}$ yields

$$
\begin{aligned}
0=\int_{0}^{t} \int_{\Omega \cap B(5 \rho)}\left\{\zeta^{2}\left(\frac{1}{2}\left[(w-k)^{+}\right]^{2}\right) t\right. & \\
& +\left[\frac{\zeta^{2} \nabla(w-k)^{+}}{M(5 \rho)+\varepsilon-u^{+}}\right. \\
& \left.+\frac{\zeta^{2}(w-k)^{+} \nabla u^{+}}{\left(M(5 \rho)+\varepsilon-u^{+}\right)^{2}}+\frac{(w-k)^{+} 2 \zeta \nabla \zeta}{M(5 \rho)+\varepsilon-u^{+}}\right] \cdot \mathbf{A} \\
& \left.+\frac{\zeta^{2}(w-k)^{+} B}{M(5 \rho)+\varepsilon-u^{+}}\right\} d x d t \\
& \quad t \in\left(0,25 \rho^{2}\right) .
\end{aligned}
$$

By virtue of the appearance of $\zeta(x)$ and $(w-k)^{+}$in (16) the effective integral domain is only

$$
\left\{\Omega \cap\left(B\left(4 \rho+\rho_{1}\right) \backslash B\left(2 \rho-\rho_{1}\right)\right) \times(0, t)\right\} \cap\{w>k\},
$$

on which $u^{+}>0$ because of (14). By the use of (2) it follows from (16) that

$$
\begin{aligned}
& \frac{1}{2} \int_{\Omega \cap B(5 \rho)} \zeta^{2}\left[(w-k)^{+}\right]^{2} d x \\
& \quad+\kappa_{0} \int_{0}^{t} \int_{\Omega \cap B(5 \rho)}\left(\zeta^{2}\left|\nabla(w-k)^{+}\right|^{2}+\zeta^{2}(w-k)^{+}\left|\nabla(w-k)^{+}\right|^{2}\right) d x d t \\
& \quad \leq \int_{0}^{t} \int_{\Omega \cap B(5 \rho)}(w-k)^{+}\left[2 \zeta|\nabla \zeta| \kappa_{1}+\zeta^{2} b(x, t)\right]\left|\nabla(w-k)^{+}\right| d x d t
\end{aligned}
$$

With the aid of Young's inequality it follows from the inequality above that

$$
\begin{aligned}
& \int_{\Omega \cap B(5 \rho)} \zeta^{2}\left[(w-k)^{+}\right]^{2} d x+\int_{0}^{t} \int_{\Omega \cap B(5 \rho)} \zeta^{2}\left|\nabla(w-k)^{+}\right|^{2} d x d t \\
& \leq C \int_{0}^{t} \int_{\Omega \cap B\left(4 \rho+\rho_{1}\right) \backslash B\left(2 \rho-\rho_{1}\right)}(w-k)^{+}\left[|\nabla \zeta|^{2}+\zeta^{2}|b(x, t)|^{2}\right] d x d t \\
& \leq C\left(\frac{1}{\left(\rho_{1}-\rho_{2}\right)^{2}}+\frac{1}{\rho^{2}}\right) \int_{0}^{t} \int_{\Omega \cap B\left(4 \rho+\rho_{1}\right) \backslash B\left(2 \rho-\rho_{1}\right)}(w-k)^{+} d x d t,
\end{aligned}
$$

where the last inequality in (18) is obtained by the fact that $(3)^{\prime}$ holds on the effective integral domain (17) and the constant $C>0$ depends 
only on $n, \kappa_{0}, \kappa_{1}$ and $K$. Extend $w$ by taking $w(x, t)=0$ as $x \notin \Omega$. We have from (4)

$$
\begin{gathered}
\operatorname{meas}\left(\left\{B\left(4 \rho+\rho_{1}\right) \backslash B\left(2 \rho-\rho_{1}\right)\right\} \cap\left\{(w-k)^{+}=0\right\}\right) \\
\geq(1-\theta) \operatorname{meas}\left\{B\left(4 \rho+\rho_{1}\right) \backslash B\left(2 \rho-\rho_{1}\right)\right\} .
\end{gathered}
$$

For $p=1,2$ applying Lemma 2 to $(w-k)^{+}$on $B\left(4 \rho+\rho_{1}\right) \backslash B\left(2 \rho-\rho_{1}\right)$, we obtain

$$
\begin{aligned}
& \int_{\Omega \cap B\left(4 \rho+\rho_{1}\right) \backslash B\left(2 \rho-\rho_{1}\right)}(w-k)^{+} d x \\
& \quad \leq C(n, \theta) \rho \int_{\Omega \cap B\left(4 \rho+\rho_{1}\right) \backslash B\left(2 \rho-\rho_{1}\right)}\left|\nabla(w-k)^{+}\right| d x
\end{aligned}
$$

and

$$
\begin{aligned}
& (19)^{\prime \prime} \quad \int_{\Omega \cap B\left(4 \rho+\rho_{1}\right) \backslash B\left(2 \rho-\rho_{1}\right)}\left[(w-k)^{+}\right]^{2} d x \\
& \quad \leq C(n, \theta) \rho^{2} \int_{\Omega \cap B\left(4 \rho+\rho_{1}\right) \backslash B\left(2 \rho-\rho_{1}\right)}\left|\nabla(w-k)^{+}\right|^{2} d x
\end{aligned}
$$

respectively. It follows from (18) and $(19)^{\prime}$ that

(20) $\int_{\Omega \cap B(5 \rho)} \zeta^{2}\left[(w-k)^{+}\right]^{2} d x+\int_{0}^{t} \int_{\Omega \cap B(5 \rho)} \zeta^{2}\left|\nabla(w-k)^{+}\right|^{2} d x d t$

$$
\begin{aligned}
\leq & C\left[\frac{1}{\left(\rho_{1}-\rho_{2}\right)^{2}}+\frac{1}{\rho^{2}}\right] \rho \\
& \cdot \int_{\Omega \cap B\left(4 \rho+\rho_{1}\right) \backslash B\left(2 \rho-\rho_{1}\right)}\left|\nabla(w-k)^{+}\right| d x d t \\
\leq & C\left[\frac{1}{\left(\rho_{1}-\rho_{2}\right)^{2}}+\frac{1}{\rho^{2}}\right]^{2} \rho^{2} \int_{\Omega \cap B\left(4 \rho+\rho_{1}\right) \backslash B\left(2 \rho-\rho_{1}\right)} \chi(k) d x d t \\
& +\frac{1}{4} \int_{\Omega \cap B\left(4 \rho+\rho_{1}\right) \backslash B\left(2 \rho-\rho_{1}\right)}\left|\nabla(w-k)^{+}\right|^{2} d x d t
\end{aligned}
$$

where the constant $C>0$ depends only on $n, \kappa_{0}, \kappa_{1}, K$ and $\theta$, and $\chi(k)$ is the characteristic function of the set $\{w>k\}$. Taking the supremum in (20) for $t \in\left(0, \rho^{2}\right)$ we get 
(21)

$$
\begin{aligned}
\underset{t \in\left(0, \rho^{2}\right)}{\operatorname{ess~sup}} & \int_{\Omega \cap B(5 \rho)} \zeta^{2}\left[(w-k)^{+}\right]^{2} d x \\
& +\int_{0}^{\rho^{2}} \int_{\Omega \cap B(5 \rho)} \zeta^{2}\left|\nabla(w-k)^{+}\right|^{2} d x d t \\
\leq & C\left[\frac{1}{\left(\rho_{1}-\rho_{2}\right)^{2}}+\frac{1}{\rho^{2}}\right] \rho^{2} \\
& \cdot \int_{0}^{\rho^{2}} \int_{\Omega \cap B\left(4 \rho+\rho_{1}\right) \backslash B\left(2 \rho-\rho_{1}\right)} \chi(k) d x d t \\
& +\frac{1}{2} \int_{0}^{\rho^{2}} \int_{\Omega \cap B\left(4 \rho+\rho_{1}\right) \backslash B\left(2 \rho-\rho_{1}\right)}\left|\nabla(w-k)^{+}\right|^{2} d x d t .
\end{aligned}
$$

According to the definition of $\zeta(x)$ it is obvious that

$$
\begin{gathered}
\int_{0}^{\rho^{2}} \int_{\Omega \cap B\left(4 \rho+\rho_{2}\right) \backslash B\left(2 \rho-\rho_{2}\right)}\left|\nabla(w-k)^{+}\right|^{2} d x d t \\
\leq \int_{0}^{\rho^{2}} \int_{\Omega \cap B(5 \rho)} \zeta^{2}\left|\nabla(w-k)^{+}\right|^{2} d x d t .
\end{gathered}
$$

On account of $C$ being independent of $\rho_{1}$ and $\rho_{2}$ and the arbitrariness of $\rho_{1}$ and $\rho_{2}$ in $0 \leq \rho_{2}<\rho_{1} \leq \rho$, combining (22) with (21) and applying Lemma 3 we obtain

$$
\begin{aligned}
\int_{0}^{\rho^{2}} & \int_{\Omega \cap B\left(4 \rho+\rho_{2}\right) \backslash B\left(2 \rho-\rho_{2}\right)}\left|\nabla(w-k)^{+}\right|^{2} d x d t \\
\leq & C\left[\frac{1}{\left(\rho_{1}-\rho_{2}\right)^{2}}+\frac{1}{\rho^{2}}\right]^{2} \rho^{2} \\
& \cdot \int_{0}^{\rho^{2}} \int_{\Omega \cap B\left(4 \rho+\rho_{1}\right) \backslash B\left(2 \rho-\rho_{1}\right)} \chi(k) d x d t,
\end{aligned}
$$

where the constant $C>0$ is independent of $\rho_{1}, \rho_{2}$ and $\rho$. Therefore, if $0 \leq \rho_{1}<\rho_{0} \leq \rho$, it follows from (23) by replacing $\rho_{1}$ and $\rho_{2}$ by $\rho_{0}$ and $\rho_{1}$ respectively that

$$
\begin{aligned}
\int_{0}^{\rho^{2}} & \int_{\Omega \cap B\left(4 \rho+\rho_{1}\right) \backslash B\left(2 \rho-\rho_{1}\right)}\left|\nabla(w-k)^{+}\right|^{2} d x d t \\
\leq & C\left[\frac{1}{\left(\rho_{0}-\rho_{1}\right)^{2}}+\frac{1}{\rho^{2}}\right]^{2} \rho^{2} \\
& \cdot \int_{0}^{\rho^{2}} \int_{\Omega \cap B\left(4 \rho+\rho_{0}\right) \backslash B\left(2 \rho-\rho_{0}\right)} \chi(k) d x d t .
\end{aligned}
$$


From (15) we have

$$
\begin{aligned}
& \left(\int_{0}^{\rho^{2}} \int_{\Omega \cap B\left(4 \rho+\rho_{2}\right) \backslash B\left(2 \rho-\rho_{2}\right)}\left|(w-k)^{+}\right|^{q} d x d t\right)^{2 / q} \\
& \leq C(n)||\left|\zeta(x)(w-k)^{+}\right| \|_{\left\{\Omega \cap B\left(4 \rho+\rho_{1}\right) \backslash B\left(2 \rho-\rho_{1}\right)\right\} \times\left(0, \rho^{2}\right)} \\
& \leq C(n)\left\{\operatorname{esssup}_{t \in\left(0, \rho^{2}\right)} \int_{\Omega \cap B(5 \rho)} \zeta^{2}\left[(w-k)^{+}\right]^{2} d x\right. \\
& +\int_{0}^{\rho^{2}} \int_{\Omega \cap B(5 \rho)} \zeta^{2}\left|\nabla(w-k)^{+}\right|^{2} d x d t \\
& \left.+\int_{0}^{\rho^{2}} \int_{\Omega \cap B\left(4 \rho+\rho_{1}\right) \backslash B\left(2 \rho-\rho_{1}\right)}|\nabla \zeta|^{2}\left|(w-k)^{+}\right|^{2} d x d t\right\} .
\end{aligned}
$$

Collecting (19)" $,(21),(24)$ and (25), it follows that

$$
\begin{aligned}
& \left(\int_{0}^{\rho^{2}} \int_{\Omega \cap B\left(4 \rho+\rho_{2}\right) \backslash B\left(2 \rho-\rho_{2}\right)}\left|(w-k)^{+}\right|^{q} d x d t\right)^{2 / q} \\
& \leq C\left[\frac{1}{\left(\rho_{1}-\rho_{2}\right)^{2}}+\frac{1}{\rho^{2}}\right]^{2} \rho^{2} \int_{0}^{\rho^{2}} \int_{\Omega \cap B\left(4 \rho+\rho_{1}\right) \backslash B\left(2 \rho-\rho_{1}\right)} \chi(k) d x d t \\
& \quad+C\left[\frac{1}{\left(\rho_{0}-\rho_{1}\right)^{2}}+\frac{1}{\rho^{2}}\right]^{2} \rho^{2} \int_{0}^{\rho^{2}} \int_{\Omega \cap B\left(4 \rho+\rho_{0}\right) \backslash B\left(2 \rho-\rho_{0}\right)} \chi(k) d x d t,
\end{aligned}
$$

where $C>0$ depends only on $n, \kappa_{0}, \kappa_{1}, K$ and $\theta$. In particular, let $0 \leq \rho^{\prime \prime}=\rho_{2}<\rho_{0}=\rho^{\prime}<\rho$ and $\rho_{1}=\frac{1}{2}\left(\rho^{\prime}+\rho^{\prime \prime}\right)$. The inequality above can be rewritten as follows:

$$
\begin{gathered}
\left(\int_{0}^{\rho^{2}} \int_{\Omega \cap B\left(4 \rho+\rho^{\prime \prime}\right) \backslash B\left(2 \rho-\rho^{\prime \prime}\right)}\left|(w-k)^{+}\right|^{q} d x d t\right)^{2 / q} \\
\leq C\left[\frac{1}{\left(\rho^{\prime}-\rho^{\prime \prime}\right)^{2}}+\frac{1}{\rho^{2}}\right]^{2} \rho^{2} \\
\cdot \int_{0}^{\rho^{2}} \int_{\Omega \cap B\left(4 \rho+\rho^{\prime}\right) \backslash B\left(2 \rho-\rho^{\prime}\right)} \chi(k) d x d t .
\end{gathered}
$$

Take for $\nu=0,1,2, \ldots$

$$
\begin{aligned}
& \rho_{\nu}=\rho / 2^{\nu}, \quad k_{\nu}=H-H / 2^{\nu} \quad(H>0 \text { will be special }), \\
& A_{\nu}=\int_{0}^{\rho^{2}} \int_{\Omega \cap B\left(4 \rho+\rho_{\nu}\right) \backslash B\left(2 \rho-\rho_{\nu}\right)} \chi\left(k_{\nu}\right) d x d t .
\end{aligned}
$$


Since the constant $C$ in (26) is independent of $\rho^{\prime}, \rho^{\prime \prime}$ and $k$, replace $\rho^{\prime}, \rho^{\prime \prime}$ by $\rho_{\nu}, \rho_{\nu+1}$, and $k$ by $k_{\nu}$, it follows from (26) that

$$
\begin{aligned}
\left(k_{\nu+1}\right. & \left.-k_{\nu}\right)^{2} A_{\nu+1}^{2 / q} \\
& \leq\left(\int_{0}^{\rho^{2}} \int_{\Omega \cap B\left(4 \rho+\rho_{\nu+1}\right) \backslash B\left(2 \rho-\rho_{\nu+1}\right)}\left|\left(w-k_{\nu}\right)^{+}\right|^{q} d x d t\right)^{2 / q} \\
& \leq C\left[\frac{1}{\left(\rho_{\nu}-\rho_{\nu+1}\right)^{2}}+\frac{1}{\rho^{2}}\right]^{2} \rho^{2} A_{\nu}, \quad \nu=0,1,2, \ldots,
\end{aligned}
$$

namely,

$$
\begin{aligned}
A_{\nu+1}^{2 / q} & \leq C\left(\frac{2^{\nu+1}}{H}\right)^{2}\left[\left(\frac{2^{\nu+1}}{\rho}\right)^{2}+\frac{1}{\rho^{2}}\right]^{2} \rho^{2} A_{\nu} \\
& \leq C 2^{8} \cdot 2^{6 \nu}(H \rho)^{-2} A_{\nu}, \quad \nu=0,1,2, \ldots .
\end{aligned}
$$

For $\nu=0$ we have

$$
A_{0}=\int_{0}^{\rho^{2}} \int_{\Omega \cap B(5 \rho) \backslash B(\rho)} \chi(0) d x d t \leq \operatorname{meas} B(5) \rho^{n+2} .
$$

As long as we assume $H>0$ so large that

$$
\begin{aligned}
\left(\frac{C \cdot 2^{8}}{H}\right)^{1+2 /(n+2)}[\operatorname{meas} B(5)]^{2 /(n+2)} \leq & \delta, \\
& 2^{6(1+2 /(n+2))} \delta^{2 /(n+2)}=1,
\end{aligned}
$$

from (27), (28) and (29) it can be shown by induction that

$$
A_{\nu} \leq \delta^{\nu} A_{0}, \quad \nu=1,2, \ldots
$$

Let $\nu \rightarrow \infty$; then

$$
\int_{0}^{\rho^{2}} \int_{\Omega \cap B(4 \rho) \backslash B(2 \rho)} \chi(H) d x d t=0,
$$

which implies

$$
\underset{\{\Omega \cap B(4 \rho) \backslash B(2 \rho)\} \times\left(0, \rho^{2}\right)}{\operatorname{ess} \sup } w \leq H .
$$

According to the definition of $w$ we have

$$
\underset{\{\Omega \cap B(4 \rho) \backslash B(2 \rho)\} \times\left(0, \rho^{2}\right)}{\operatorname{ess} \sup } u^{+} \leq[M(5 \rho)+\varepsilon]\left(1-e^{-H}\right) .
$$

Let $\varepsilon \rightarrow 0$; then

$$
\underset{\{\Omega \cap B(4 \rho) \backslash B(2 \rho)\} \times\left(0, \rho^{2}\right)}{\operatorname{ess} \sup ^{+}} u^{+} \leq M(5 \rho)\left(1-e^{-H}\right) .
$$


It follows from Lemma 1 that

$$
\begin{aligned}
M(\rho) & =\underset{\{\Omega \cap B(\rho)\} \times\left(0, \rho^{2}\right)}{\text { ess sup }} u \leq \underset{\{\Omega \cap B(3 \rho)\} \times\left(0, \rho^{2}\right)}{\operatorname{ess} \sup } u \\
& \leq \underset{\{\Omega \cap B(4 \rho) \backslash B(2 \rho)\} \times\left(0, \rho^{2}\right)}{\operatorname{ess} \sup } u^{+} \leq M(5 \rho)\left(1-e^{-H}\right) .
\end{aligned}
$$

We see from (29) that $H$ is determined by constants $C$ and $n$; hence, $H$ is independent of $\rho$.

Now, suppose $\rho_{0}=\max (R, 1)$. For any $\rho \geq \rho_{0}$ there exists an integer $\nu$ such that $5^{\nu} \rho_{0} \leq \rho<5^{\nu+1} \rho_{0}$. Iterating by (30) we get

$$
\begin{aligned}
M(\rho) \geq & M\left(5^{\nu} \rho_{0}\right) \geq\left(1-e^{-H}\right)^{-\nu} M\left(\rho_{0}\right) \\
\geq & \left(1-e^{-H}\right) M\left(\rho_{0}\right)\left(1-e^{-H}\right)^{-\log _{5}\left(\rho / \rho_{0}\right)} \\
= & \left(1-e^{-H}\right) M\left(\rho_{0}\right)\left(\rho / \rho_{0}\right)^{\lambda} \geq\left(1-e^{-H}\right) M(R)\left(\rho / \rho_{0}\right)^{\lambda}, \\
& \quad \lambda=\log _{5}\left(1-e^{-H}\right)^{-1}>0, \quad \rho \geq \rho_{0} .
\end{aligned}
$$

Thus, $M(\rho) \rightarrow \infty$ as $\rho \rightarrow \infty$ whenever $M(R)>0$. The proof of the theorem is completed.

\section{REFERENCES}

[1] S. Granlund, A Phragmén-Lindelöf principle for subsolutions of quasi-linear equations, Manuscripta Math., 36 (1981), 355-365.

[2] P. Tolksdorf, On the Dirichlet problem for quasilinear equations in domains with conical boundary points, Comm. Partial Differential Equations, 8 (1983), 773-817.

[3] P. Lindqvist, On the growth of the solutions of the differential equation $\operatorname{div}\left(|\nabla u|^{p-2} \nabla u\right)=0$ in $n$-dimensional space, J. Differential Equations, 58 (1985), 307-317.

[4] Xi-ting Liang, The Phragmén-Lindelöf principle for generalized solutions of quasi-linear elliptic equations, J. Chengdu Univ. (Natur. Sci.) 5, 1 (1986), 1-7 (in Chinese).

[5] P. Aviles, Phragmén-Lindelöf theorems for non-linear elliptic equations, Arch. Rational Mech. Anal., 97 (1987), 141-170.

[6] Xi-ting Liang, $A$ behavior for solutions of parabolic equations, Acta Math. Sci., 9 (1989), 147-153 (in Chinese).

[7] J. Moser, A new proof of de Giorgi's Theorem concerning the regularity problem for elliptic differential equations, Comm. Pure Appl. Math., 13 (1960), 457-468.

[8] O. A. Ladyženskaja and N. N. Ural'ceva, On the Hölder continuity of solutions and their derivatives for linear and quasilinear equations of elliptic and parabolic types, Dokl. Akad. Nauk SSSR, 155 (1964), 1258-1261 (in Russian).

[9] C. B. Morrey, Multiple Integrals in the Calculus of Variations, Springer-Verlag, New York, 1966.

[10] M. Giaquinta and E. Giusti, On the regularity of the minima of variational integrals, Acta Math., 148 (1982), 31-46. 
[11] O. A. Ladyženskaja, V. A. Solonnikov and N. N. Ural'ceva, Linear and quasilinear equations of parabolic type, Transl. Math. Monographs, vol. 23, Amer. Math. Soc., Providence, R.I. 1968.

Received September 28, 1990.

ZHONGSHAN UNIVERSITY

GUANGZHOU, 510275

People's RePublic of China

AND

TIANJIN NORMAL UNIVERSITY

TIANJIN, 300073

People's Republic of China 



\section{PACIFIC JOURNAL OF MATHEMATICS EDITORS}

\author{
V. S. VARADARAJAN \\ (Managing Editor) \\ University of California \\ Los Angeles, CA 90024-1555-05 \\ Herbert Clemens \\ University of Utah \\ Salt Lake City, UT 84112 \\ F. Michael Christ \\ University of California \\ Los Angeles, CA 90024-1555 \\ THOMAS ENRIGHT \\ University of California, San Diego \\ La Jolla, CA 92093
}

Nicholas ERCOLANI

University of Arizona

Tucson, AZ 85721

R. FINN

Stanford University

Stanford, CA 94305

VAUGHAN F. R. JONES

University of California

Berkeley, CA 94720

Steven Kerckhoff

Stanford University

Stanford, CA 94305

\section{C. MOORE}

University of California

Berkeley, CA 94720

Martin ScharlemanN

University of California

Santa Barbara, CA 93106

HAROLD STARK

University of California, San Diego

La Jolla, CA 92093

\section{R. ARENS \\ ASSOCIATE EDITORS}

\begin{tabular}{|c|c|c|c|c|}
\hline R. ARENS & $\begin{array}{l}\text { E. F. BECKENBACH } \\
(1906-1982)\end{array}$ & B. H. NeumanN & $\begin{array}{c}\text { F. WolF } \\
(1904-1989)\end{array}$ & K. YoshidA \\
\hline \multicolumn{5}{|c|}{ SUPPORTING INSTITUTIONS } \\
\hline \multicolumn{2}{|c|}{ UNIVERSITY OF ARIZONA } & UNIVER: & OF OREG & \\
\hline \multicolumn{2}{|c|}{ UNIVERSITY OF BRITISH COLUMB } & & & \\
\hline \multicolumn{2}{|c|}{ CALIFORNIA INSTITUTE OF TECHNOLOGY } & OGY STANI & INIV & \\
\hline \multicolumn{2}{|c|}{ UNIVERSITY OF CALIFORNIA } & UNI & & \\
\hline \multicolumn{2}{|c|}{ MONTANA STATE UNIVERSITY } & UNIVE & OF T & \\
\hline \multirow{2}{*}{\multicolumn{2}{|c|}{ NIVERSITY OF NEVADA, RENO }} & UNIVE & OF U & \\
\hline & & WASH & N ST & ERSIT \\
\hline \multicolumn{2}{|c|}{$\begin{array}{l}\text { NEW MEXICO STATE UNIVERSITY } \\
\text { OREGON STATE UNIVERSITY }\end{array}$} & UNIVERS & OF WASHI & \\
\hline
\end{tabular}

The Supporting Institutions listed above contribute to the cost of publication of this Journal, but they are not owners or publishers and have no responsibility for its content or policies.

Mathematical papers intended for publication in the Pacific Journal of Mathematics should be in typed form or offset-reproduced (not dittoed), double spaced with large margins. Please do not use built up fractions in the text of the manuscript. However, you may use them in the displayed equations. Underline Greek letters in red, German in green, and script in blue. The first paragraph must be capable of being used separately as a synopsis of the entire paper. In particular it should contain no bibliographic references. Please propose a heading for the odd numbered pages of less than 35 characters. Manuscripts, in triplicate, may be sent to any one of the editors. Please classify according to the 1991 Mathematics Subject Classification scheme which can be found in the December index volumes of Mathematical Reviews. Supply name and address of author to whom proofs should be sent. All other communications should be addressed to the managing editor, or Elaine Barth, University of California, Los Angeles, California 90024-1555-05.

There are page-charges associated with articles appearing in the Pacific Journal of Mathematics. These charges are expected to be paid by the author's University, Government Agency or Company. If the author or authors do not have access to such Institutional support these charges are waived. Single authors will receive 50 free reprints; joint authors will receive a total of 100 free reprints. Additional copies may be obtained at cost in multiples of 50 .

The Pacific Journal of Mathematics (ISSN 0030-8730) is published monthly except for July and August. Regular subscription rate: $\$ 190.00$ a year (10 issues). Special rate: $\$ 95.00$ a year to individual members of supporting institutions.

Subscriptions, orders for numbers issued in the last three calendar years, and changes of address should be sent to Pacific Journal of Mathematics, P.O. Box 969, Carmel Valley, CA 93924, U.S.A. Old back numbers obtainable from Kraus Periodicals Co., Route 100, Millwood, NY 10546.

The Pacific Journal of Mathematics at P.O. Box 969, Carmel Valley, CA 93924 (ISSN 0030-8730) is published monthly except for July and August. Second-class postage paid at Carmel Valley, California 93924, and additional mailing offices. Postmaster: send address changes to Pacific Journal of Mathematics, P.O. Box 969, Carmel Valley, CA 93924.

PUBLISHED BY PACIFIC JOURNAL OF MATHEMATICS, A NON-PROFIT CORPORATION Copyright (C) 1992 by Pacific Journal of Mathematics 


\section{Pacific Journal of Mathematics}

Vol. 153, No. $2 \quad$ April, 1992

R. Ayala, Eladio Domínguez Murillo, Alberto Márquez Pérez and A.

Quintero, Lusternik-Schnirelmann invariants in proper homotopy

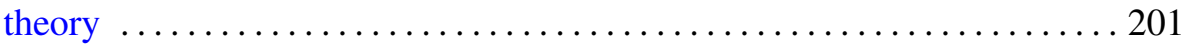

Hari Bercovici and Dan-Virgil Voiculescu, Lévy-Hinčin type theorems for multiplicative and additive free convolution $\ldots \ldots \ldots \ldots \ldots \ldots \ldots 217$

L. J. Bunce and Cho-Ho Chu, Compact operations, multipliers and Radon-Nikodým property in $J B^{*}$-triples $\ldots \ldots \ldots \ldots \ldots \ldots \ldots \ldots \ldots \ldots \ldots \ldots$

Marius Dadarlat, Gabriel Nagy, András Némethi and Cornel Pasnicu, Reduction of topological stable rank in inductive limits of $C^{*}$-algebras

François Dumas and Robert Vidal, Dérivations, et hautes dérivations, dans certains corps gauches de series de Laurent .................... 277

Mourad Ismail and Xin Li, On sieved orthogonal polynomials. IX:

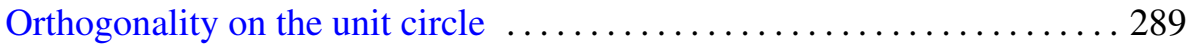

X. T. Liang and Y. W. Lu, A Phragmén-Lindelöf theorem ...............299

Mark Stephen Reeder, On certain Iwahori invariants in the unramified principal series

Shohei Tanaka, On the representation of the determinant of Harish-Chandra's $C$-function of $\operatorname{SL}(n, \mathbb{R})$

Fritz von Haeseler and Guentcho Svetoslavov Skordev, Borsuk-Ulam theorem, fixed point index and chain approximations for maps with multiplicity 